\title{
Path Loss Characterization of Horn-to-Horn and Textile-to-Textile On-Body mmWave Channels at $60 \mathrm{GHz}$
}

\author{
Mouad Ghandi $^{1}$, Emmeric Tanghe ${ }^{2}$, Wout Joseph ${ }^{2}$, Mustapha Benjillali ${ }^{3}$ and Zouhair Guennoun ${ }^{1}$ \\ ${ }^{1}$ Laboratory of Electronics and Communications, EMI, Mohammed V University, Rabat, Morocco \\ ${ }^{2}$ iMinds - Department of Information Technology, Ghent University, Ghent, Belgium \\ ${ }^{3}$ Communications Systems Department, INPT, Rabat, Morocco \\ Emails: mouad.ghandi.92@gmail.com, \{emmeric.tanghe, wout.joseph $\}$ intec.ugent.be \\ benjillali@ieee.org, zouhair@emi.ac.ma
}

\begin{abstract}
Wireless body area networks have raised considerable attention within the wireless personal area community. To optimize the network architecture and the energy consumption of on-body equipments, a good knowledge and understanding of the radio link and the propagation channel are required. This work addresses the influence of medium presence on the reflection loss of horn and textile antennas. In particular, the path loss in free space and under skin-equivalent phantom of horn-to-horn and textile-to-textile communications is presented. The obtained results show that the path loss differs considerably in the analyzed situations; with horn-to-horn links showing a path loss exponent between 1.13 and 2.35 , while it is reaching 4.7 for textile-totextile links. The obtained results provide additional input for the design of communications links in the context of future 5G and IoT eco-systems.
\end{abstract}

\section{INTRODUCTION}

Body area networks (BAN) consist of a number of nodes and units placed on the human body or in close proximity such as daily wearable and accessories. BANs rely, theoretically, on Norton's investigation of radio communication using small antennas [1]. An increasing number of research works deals with the development of mmWave BANs, identified as a highly attractive solution for future wireless BAN (WBANs), with a strong potential in health care environments, entertainment, identification systems, sports, smart homes, space and military applications [2]-[4].

In the last decade, the $60 \mathrm{GHz}$ technology has attracted a huge amount of interest due to its various advantages compared to lower frequencies [5], [6]. In this unlicensed frequency range, a 7 to $9 \mathrm{GHz}$ bandwidth is typically available depending on the country. The advantages of the $60 \mathrm{GHz}$ band include high-speed links for wireless personal area networks (WPANs) and wireless local area networks (WLANs) and future WBANs, more compact systems compared to lower frequencies, and higher levels of security. Propagation in the $60 \mathrm{GHz}$ band is characterized by a high atmospheric attenuation due to the free space loss and resonant oxygeninduced absorption. On the other hand, the interaction between the human body and the millimeter waves is characterized by a relatively high reflection, due to the contrast between the dielectric permittivity of the skin and the free space. Furthermore, since the penetration depth at $60 \mathrm{GHz}$ is around $0.5 \mathrm{~mm}$, the penetration is mainly limited to the superficial layers of the human skin [7].

In this work, two types of mmWave antennas (namely, horn and textile antennas [8]) are considered. In particular, we investigate the influence of the skin-equivalent phantom on the antenna parameters for different antenna-phantom distances. In addition, the path loss (PL) in free space and along homogeneous and layered mediums of the horn-to-horn and textileto-textile communications is analyzed and characterized. Both measurements and simulation results are presented to illustrate the analysis.

The paper is organized as follows. Section II presents the characteristics of the considered antennas, and the influence of the medium on the antenna parameters is discussed in Section III. In Section IV, the path loss characterization of hornto-horn and textile-to-textile communications is presented in details, based on simulations and measurements, and conclusions are drawn in Section V.

\section{Antennas DESign}

\section{A. Horn Antenna Design}

The Horn antenna used in measurements and simulations consists of a $22 \times 16 \mathrm{~mm}$ external aperture and a length of $74 \mathrm{~mm}$ (cf. Fig. 1 (a) and (b)). Its gain in free space is $19.5 \mathrm{dBi}$, and the $-10 \mathrm{~dB}$ bandwidth is $10 \mathrm{GHz}$ centered around $60 \mathrm{GHz}$. Fig. 2 highlights the comparison between measured and simulated reflection losses in free space.

To model the source, we used the wave-guide source proposed by SEMCADx. We note that this source does not take into consideration the connector effect, which explains the difference that some perturbations/fluctuations can be observed in measurements while they are absent in simulation. In addition, in real laboratory conditions where the free space is not perfect, a slight difference with simulation is normally expected, and it is expected to increase when operating at high frequencies with small antennas. 


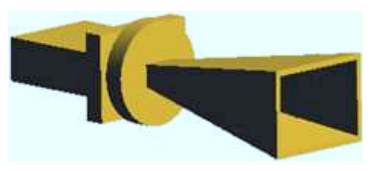

(a)

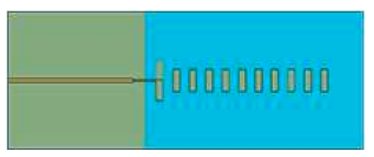

(c)

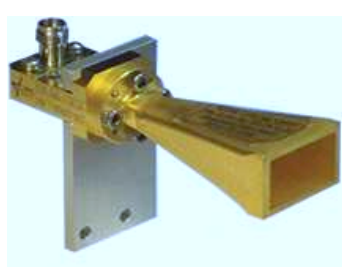

(b)

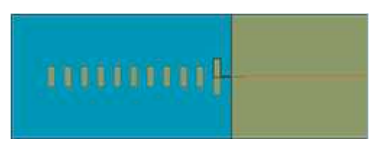

(d)
Fig. 1. Adopted and realized antenna models: (a) The horn antenna modeled with SEMCADx, (b) the horn antenna used in measurements, (c) the front side of the textile antenna, (d) the back side of the textile antenna.

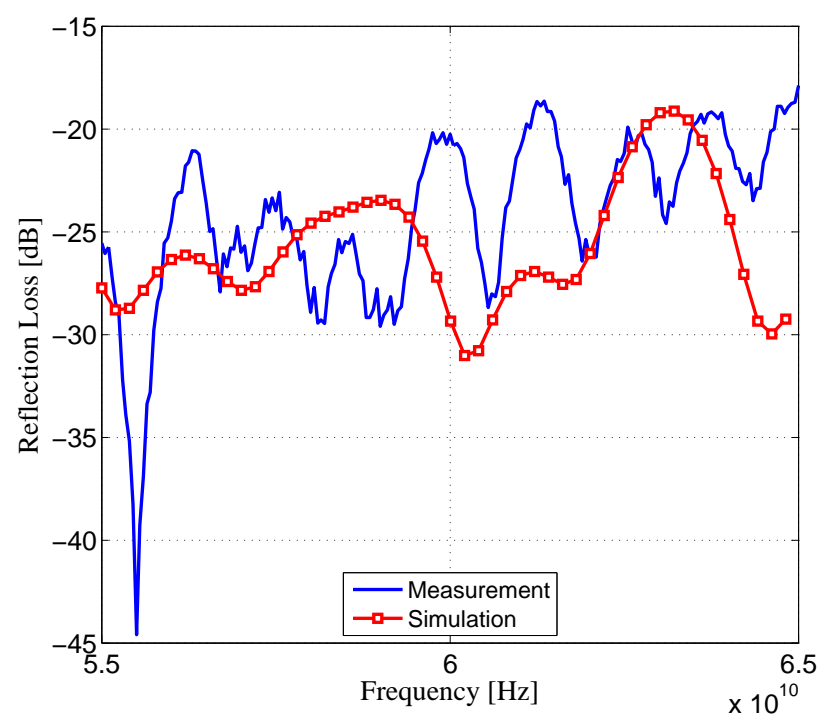

Fig. 2. The horn antenna reflection loss.

\section{B. Textile Antenna Design}

A more practical antenna for on-body communications has been presented by the authors in [8], where the direction of maximum radiation is parallel to the body surface to reduce the off-body radiation, and hence minimizing the interference between neighboring BANs. In our work, we modeled this antenna using the FDTD solver Sim4Life (S4L) as shown in Fig. 1 (c) and (d); the interested reader can refer to [8] for more details. The antenna structure is made of a driven dipole and 10 directors printed on the top layer of the textile substrate. The microstrip truncated ground plane is located at $1 \mathrm{~mm}$ from the driving dipole and acts as a reflector.

We extracted the reflection loss $S_{11}$ in free space using S4L. As illustrated in Fig. 3, the available bandwidth under $-10 \mathrm{~dB}$ is $2 \mathrm{GHz}$ around $58.86 \mathrm{GHz}$. In addition, a gain of approximately $10 \mathrm{dBi}$ at the center frequency is obtained.

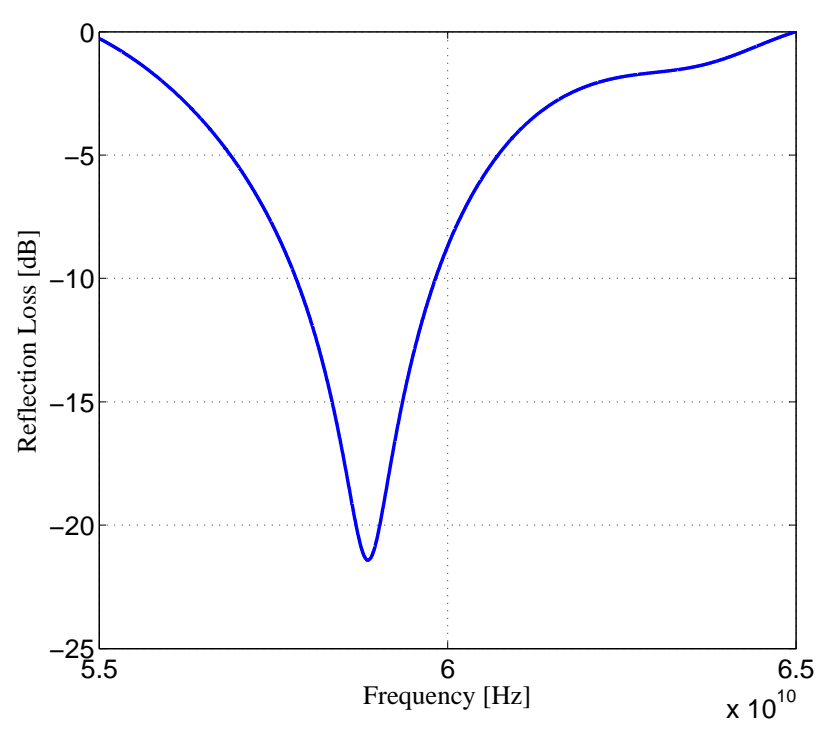

Fig. 3. Textile antenna reflection loss.

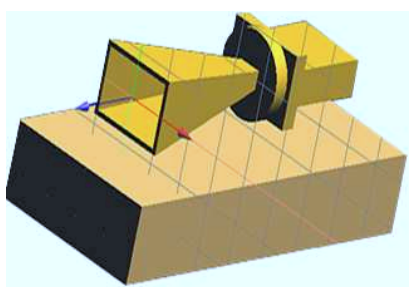

(a)

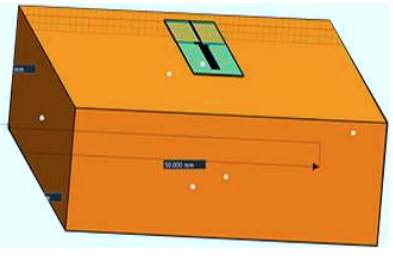

(b)
Fig. 4. Simulation setups of the antenna versus phantom for: (a) the horn antenna with SEMCADx, (b) the textile antenna with S4L.

\section{Medium InfLuence}

In this section, we present the computed reflection coefficients of both horn and textile antennas mounted on a parallepipedic skin-equivalent phantom. As depicted by Fig. 4, we used a $50 \times 100 \times 17 \mathrm{~mm}$ phantom for the horn antenna simulations, while a $50 \times 50 \times 17 \mathrm{~mm}$ phantom is used for the textile antenna. In both cases, the dielectric properties of the phantom are $\epsilon_{r}=7.89$ and $\sigma=36.4 \mathrm{~S} / \mathrm{m}$.

Fig. 5 shows the simulation results of the phantom presence effect on the horn antenna reflection loss, for different separation distances between the antenna and the phantom. It is important to notice that, for all separation distances, the influence is very similar in behavior, and although the separation distances provides different reflection losses below $59 \mathrm{GHz}$; the results become almost identical beyond that frequency. This is an important observation that will facilitate the characterization of the analyzed setup. In addition, and more importantly, the available bandwidth is still about $10 \mathrm{GHz}$ [55 GHz $-65 \mathrm{GHz}$ ] for all illustrated separation distances.

Similarly, Fig. 6 presents the reflection loss of the textile antenna when placed at various distances from the phantom. 


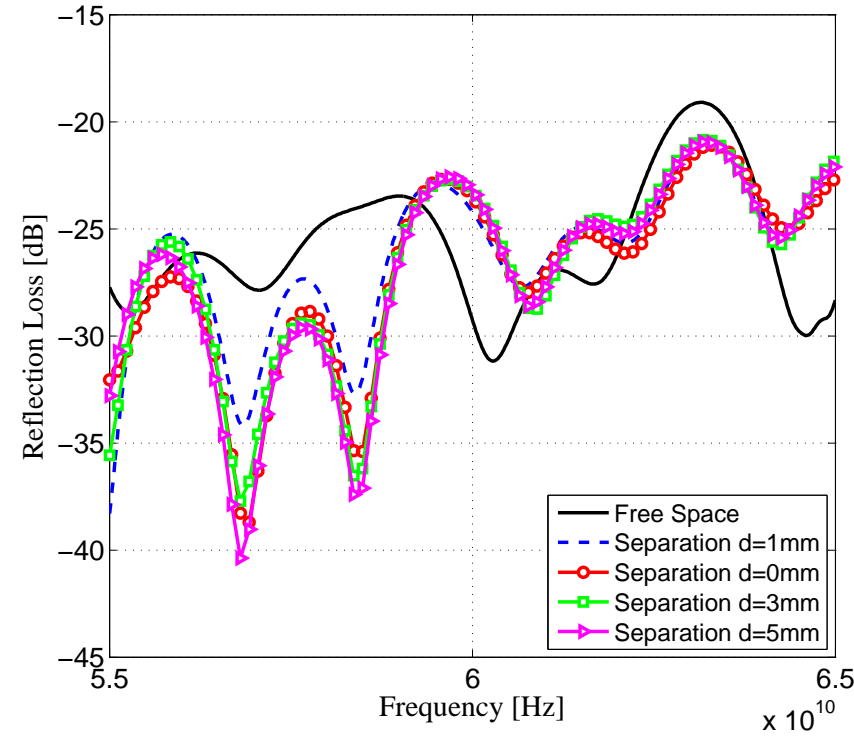

Fig. 5. Phantom presence effect on the horn antenna reflection loss for different antenna-phantom separation distances.

The results show a detuning from the free space resonance, which can be explained by the change of the antenna's effective length caused by the presence of the skin-equivalent phantom with a varying dielectric constant. As a confirmation, the antenna yields the worst performance when the antenna is directly on the phantom (i.e., no separation).

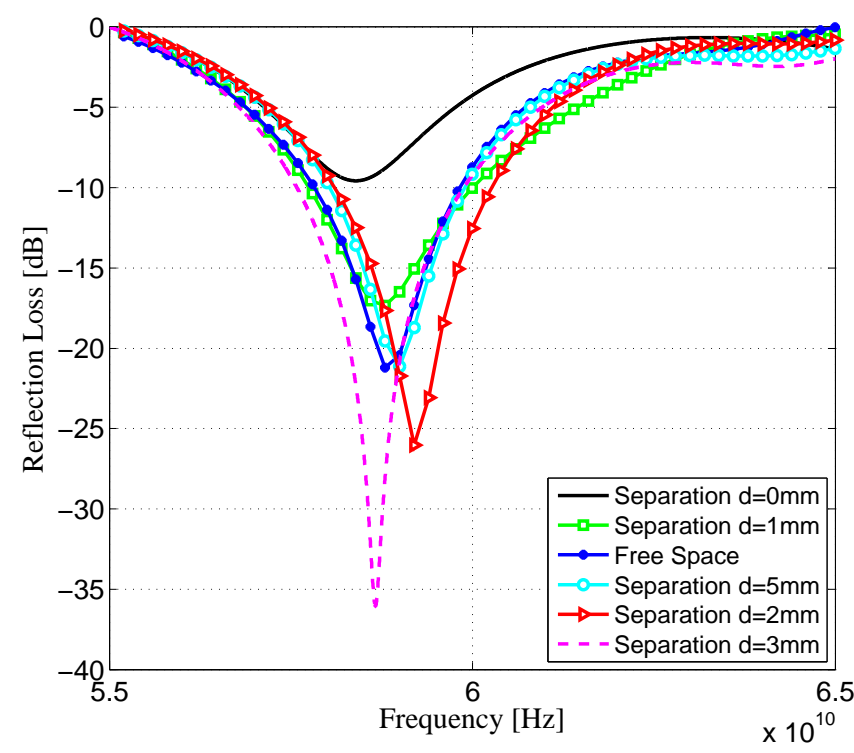

Fig. 6. Phantom presence effect on the textile antenna reflection loss for different antenna-phantom separation distances.

Table I summarizes the parameters of the reflection loss with respect to the antenna-phantom separation. At a $1 \mathrm{~mm}$ separation, the antenna shows a slight detuning from free space resonance in comparison to other separations, with a bandwidth of $2.25 \mathrm{GHz}$ that includes the $60 \mathrm{GHz}$ frequency. In
TABLE I

PARAMETERS OF THE REFLECTION LOSS WITH RESPECT TO THE ANTENNA-PHANTOM SEPARATION

\begin{tabular}{ccccc}
\hline Separation & $\mathbf{F}_{\min }(\mathbf{G H z})$ & $\mathbf{F}_{\max }(\mathbf{G H z})$ & $\mathbf{B W}(\mathbf{G H z})$ & $\mathbf{F}_{\mathbf{c}}(\mathbf{G H z})$ \\
\hline Free Space & 57.83 & 59.83 & 2 & 58.86 \\
$0 \mathrm{~mm}$ & 58.43 & 58.43 & 0 & 58.43 \\
$1 \mathrm{~mm}$ & 57.75 & 60 & 2.25 & 58.74 \\
$2 \mathrm{~mm}$ & 58.11 & 60.26 & 2.15 & 59.23 \\
$3 \mathrm{~mm}$ & 57.58 & 59.88 & 2.3 & 58.67 \\
$5 \mathrm{~mm}$ & 58.04 & 59.89 & 1.85 & 58.97 \\
\hline
\end{tabular}

the remainder, we use a $1 \mathrm{~mm}$ distance as an antenna/medium separation distance for textile-to-textile communications.

\section{PATH LOSS MODEL}

To model the path loss between the transmitting and the receiving antennas as a function of distance, we use the following semi-empirical formula, expressed in decibel and based on the Friis formula

$$
P_{\mathrm{dB}}(d)=P_{\mathrm{dB}}\left(d_{0}\right)+10 \cdot n \cdot \log \left(\frac{d}{d_{0}}\right)=-\left|S_{21}\right|_{\mathrm{dB}},
$$

where $d$ is the antennas separation expressed in centimeters, $P_{\mathrm{dB}}\left(d_{0}\right)$ is the path loss in $\mathrm{dB}$ at a reference distance $d_{0}(10$ $\mathrm{cm}$ in this work), and $n$ is the path loss exponent. The path loss is equal to $-\left|S_{21}\right|_{\mathrm{dB}}$ when the transmitting antenna has an input impedance of $Z_{\text {in }}=50 \Omega$, and the receiver is ended with an impedance $Z_{\text {out }}=50 \Omega$.

\section{A. Horn-to-Horn Model}

1) Configuration: Two identical horn antennas (cf. subsection II-A) are used at the transmitter and the receiver for both measurements and simulations, as shown on Fig. 7 (a). In all scenarios, the antennas remain parallel to each other. To investigate the on-body communication channel, we first consider the wireless channel with no separation between the skin and antennas. The distance between the antennas varies from 5 to $30 \mathrm{~cm}$ in individual steps of $2.5 \mathrm{~cm}$.

For the measurements along the stretched arm, the transmitter is positioned at the wrist and the receiver is moving over several positions towards the shoulder, while the antennas are perpendicular to the human skin. On the other hand, the onbody channel has been simulated for both "homogeneous" and "layered" flat phantoms (cf. Fig. 7 (b) and (c)).

2) Simulated PL Model: The path loss along homogeneous and layered mediums is numerically investigated using the finite-difference time domain (FDTD) solver SEMCADX. Fig. 8 shows the obtained path loss values along with the fitted model, along a homogeneous and layered mediums, as a function of the antennas separation in free space. Based on the model in (1), the parameter values of the fitted simulation models are provided in Table II.

It is clear that the path loss increases with distance as expected, with a fading variance around the mean. Also, we can note that the presence of other factors-including losses of 


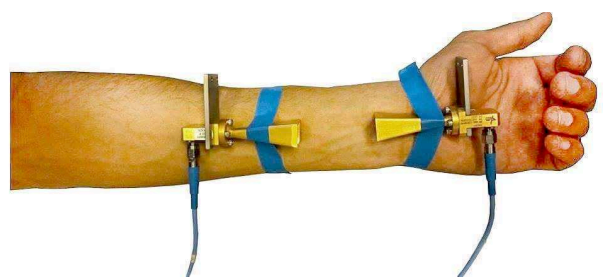

(a)

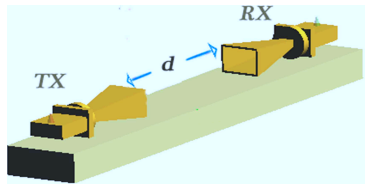

(b)

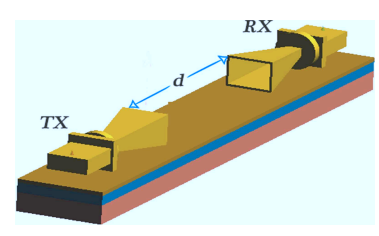

(c)
Fig. 7. Measurements and simulation settings along a stretched arm: (a) Real human arm, (b) Homogeneous medium [Muscle] (c) Layered medium [skin, fat and muscle].

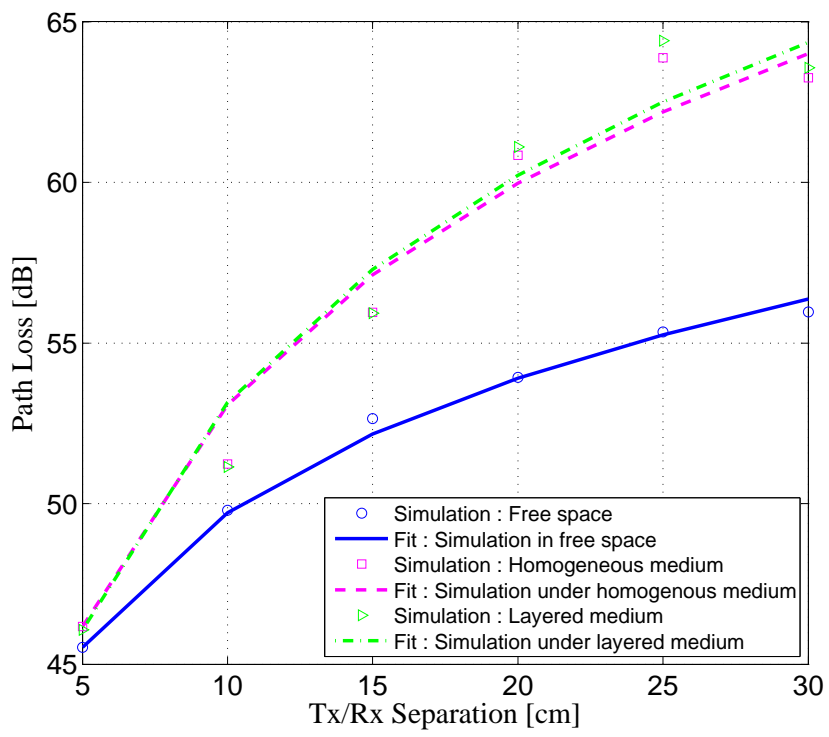

Fig. 8. Simulated fitted path loss models versus antennas separation in free space, for both homogeneous and layered mediums.

skin equivalent tissue and creeping waves for communication under flat phantoms-yields higher exponent values compared to the free space case.

The skin depth can be defined as the distance over which the amplitude of the current density vector decreases to $1 / e$ of its initial value, and it is around $0.5 \mathrm{~mm}$ at $60 \mathrm{GHz}$. This is sufficiently small compared to the skin thickness. This also explains the noticeable similarity between the homogeneous and the layered mediums parameters. Hence, the $60 \mathrm{GHz}$ model of the human body does not have to take into account the presence of underlying tissues, since the skin penetrating field is sufficiently attenuated.

3) Measured PL Model: In this subsection, the measured path loss along the arm is investigated. Fig. 9 illustrates the measured path loss versus antennas separation long the arm
TABLE II

PARAMETERS OF THE SIMULATED PATH LOSS OF HORN-TO-HORN COMMUNICATIONS IN FREE SPACE, FOR HOMOGENEOUS AND LAYERED MEDIUMS

\begin{tabular}{cccc}
\hline Parameters & Free Space) & Homogeneous Med. & Layered Med. \\
\hline$P_{\mathrm{dB}}\left(d_{0}\right)$ & 45.52 & 46.18 & 46.08 \\
$\mathrm{n}[-]$ & 1.4 & 2.29 & 2.35 \\
$\sigma[d B]$ & 0.26 & 1.22 & 1.35 \\
\hline
\end{tabular}

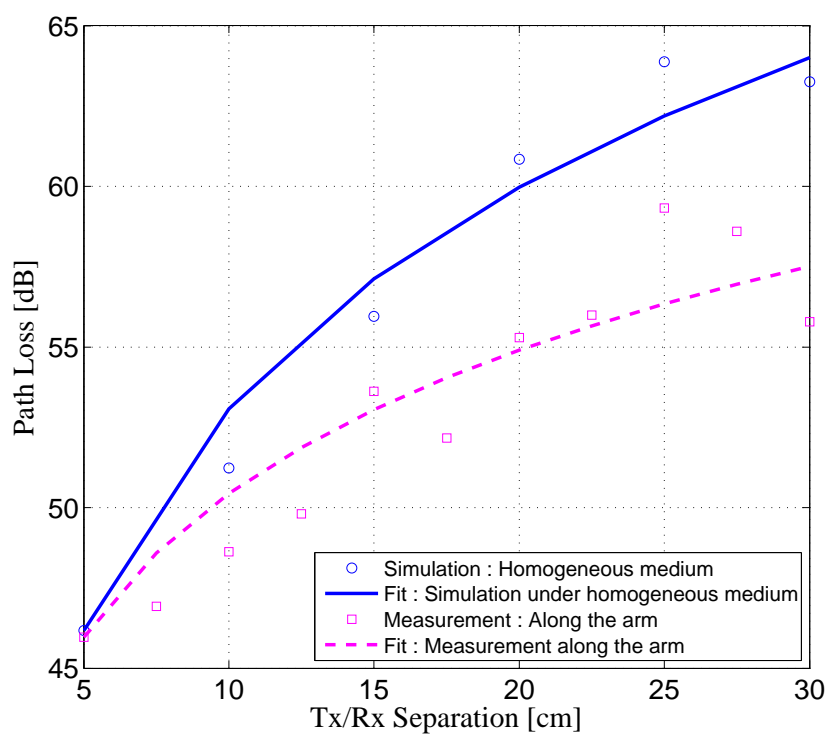

Fig. 9. Simulated and measured path loss values and fitted models vs antenna separation under homogeneous medium and along the arm, respectively.

TABLE III

PARAMETERS OF THE PATH LOSS OF HORN-TO-HORN COMMUNICATIONS ALONG THE ARM AND UNDER A HOMOGENEOUS MEDIUM

\begin{tabular}{ccc}
\hline Parameters & Along arm & Homogeneous Med. \\
\hline$P_{d B}\left(d_{0}\right)$ & 51.26 & 46.18 \\
$\mathrm{n}[-]$ & 1.13 & 2.29 \\
$\sigma[d B]$ & 2.04 & 1.22 \\
\hline
\end{tabular}

where the antennas are kept perpendicular to body surface (in contrast with the simulated path loss where antennas are parallel to the medium, cf. Fig. 8). The markers indicate the measured values, while the lines represent the path loss model obtained via a curve fitting of the measurement data.

Table III presents the model parameter values of the fitted model and the simulated model under a homogeneous medium. In measurements, the fading variance is larger because of the multipath environment nature. The path loss exponent is reduced to 1.13 , which slows the energy reduction. Subsequently, the perpendicular position, where the E-Field is normal to the body surface, would enhance the on-body communication substantially. 


\section{B. Textile-to-Textile Model}

Again, and similarly to the previous above presented setup, we re-investigate the communication link with two identical textile antennas (cf. subsection II-B) are used at the transmitter and the receiver. The gain in free space is about $10.7 \mathrm{dBi}$ for both antennas, and the $-10 \mathrm{~dB}$ bandwidth of $\left|S_{11}\right|$ is $2.15 \mathrm{GHz}$.

In all simulations, the antennas remain parallel to each other. To investigate the channel under a flat phantom, we analyze the wireless channel for a $2 \mathrm{~mm}$ separation between the skin and the antennas.

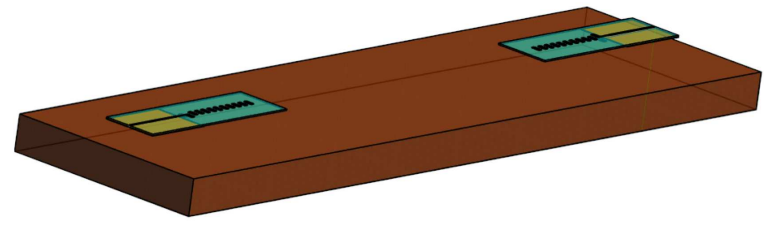

Fig. 10. Simulated textile to-textile communication under a homogeneous medium.

The path loss along an homogeneous medium is numerically investigated using the FDTD solver S4L. Fig. 11 shows the simulated path loss values along with the fitted model, both versus the antennas separation, in free space and along a homogeneous medium. Once again, and based on (1), we obtain the parameters values of the fitted model are illustrated in Table IV.

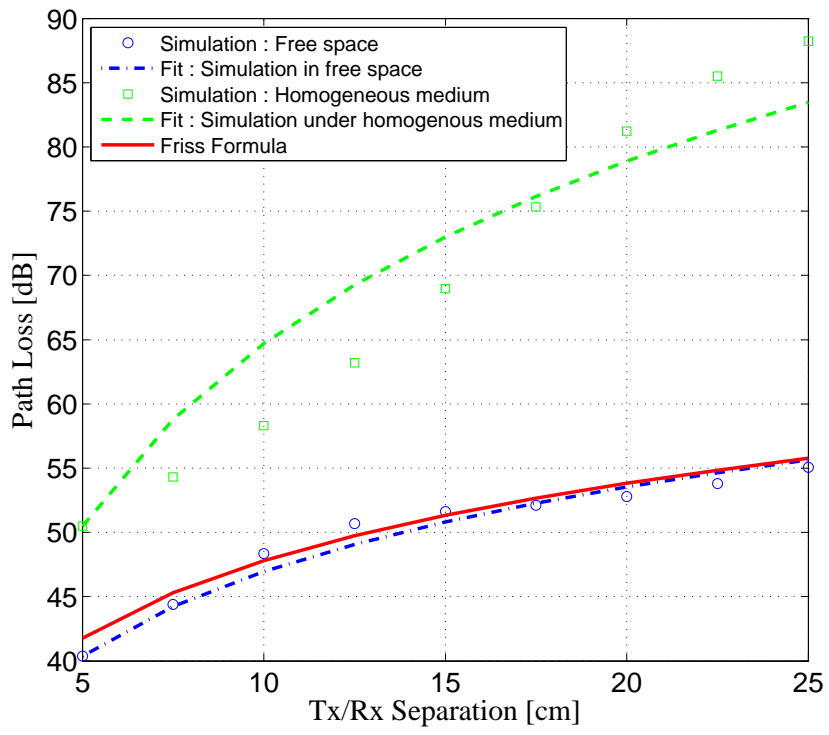

Fig. 11. Simulated path loss values and fitted models, as a function of the antennas separation, in free space and under an homogeneous medium. Results based on Friss equation are shown as a reference.

We note an agreement between the free space results and Friss formula in the far field, while in the near field; where Friss formula is not valid, there is a slight difference. Compared to the horn antenna, the energy reduction for textile-totextile communications is faster with a path loss exponent of up to 4.7 , and a high fading variance of approximately $4.2 \mathrm{~dB}$.
TABLE IV

PARAMETERS OF THE PATH LOSS OF A TEXTILE-TO-TEXTILE COMMUNICATION UNDER AN HOMOGENEOUS MEDIUM

\begin{tabular}{ccc}
\hline Parameters & Free Space & Homogeneous Med. \\
\hline$P_{d B}\left(d_{0}\right)$ & 40.37 & 50.5 \\
$\mathrm{n}[-]$ & 2.19 & 4.7 \\
$\sigma[d B]$ & 0.88 & 4.2 \\
\hline
\end{tabular}

For the textile antenna, the flat phantom presence has drastic consequences on the antenna parameters and performance, such as the detuning from the free space resonance, and the changes in the antenna's effective length caused by the medium presence.

\section{CONCLUSION}

In this work, we have studied the influence of medium presence on the reflection loss of horn and textile antennas in the context of on-body mmWave communications. The obtained results indicate that textile antennas show a significant performance impact compared to horn antennas which are, on the other hand, less practical for WBAN daily applications. Under the same conditions, the results indicate that the best antenna-phantom separation is $1 \mathrm{~mm}$, where the bandwidth is $2.25 \mathrm{GHz}$.

In addition, the path loss in free space and under skinequivalent phantom for horn-to-horn and textile-to-textile communications was also presented. The results indicate that the path loss differs considerably in the analyzed situations; with horn-to-horn links showing a path loss exponent between 1.13 and 2.35 , while it is reaching 4.7 for textile-to-textile links.

\section{REFERENCES}

[1] K. A. Norton, "The propagation of radio waves over the surface of the earth and in the upper atmosphere," Proceedings of the Institute of Radio Engineers, vol. 24, no. 10, pp. 1367-1387, Oct 1936.

[2] M. Zhadobov, N. Chahat, R. Sauleau, C. Le Quement, and Y. Le Drean, "Millimeter-wave interactions with the human body: state of knowledge and recent advances," International Journal of Microwave and Wireless Technologies, vol. 3, pp. 237-247, Apr. 2011.

[3] N. Chahat, G. Valerio, M. Zhadobov, and R. Sauleau, "On-body propagation at $60 \mathrm{GHz}, "$ IEEE Transactions on Antennas and Propagation, vol. 61, no. 4, pp. 1876-1888, Apr. 2013.

[4] Y. I. Nechayev, X. Wu, C. C. Constantinou, and P. S. Hall, "Millimetrewave path-loss variability between two body-mounted monopole antennas," IET Microwaves, Antennas Propagation, vol. 7, no. 1, pp. 1-7, Jan. 2013.

[5] P. Smulders, "Exploiting the $60 \mathrm{GHz}$ band for local wireless multimedia access: prospects and future directions," IEEE Communications Magazine, vol. 40, no. 1, pp. 140-147, Jan. 2002.

[6] R. C. Daniels and R. W. Heath Jr., "60 GHz wireless communications: emerging requirements and design recommendations," IEEE Vehicular Technology Magazine, vol. 2, no. 3, pp. 41-50, Sep. 2007.

[7] N. Chahat, M. Zhadobov, R. Sauleau, and S. I. Alekseev, "New method for determining dielectric properties of skin and phantoms at millimeter waves based on heating kinetics," IEEE Transactions on Microwave Theory and Techniques, vol. 60, no. 3, pp. 827-832, Mar. 2012.

[8] N. Chahat, M. Zhadobov, L. Le Coq, and R. Sauleau, "Wearable endfire textile antenna for on-body communications at $60 \mathrm{GHz}$," IEEE Antennas and Wireless Propagation Letters, vol. 11, no. 7, pp. 799-802, Jul. 2012. 